RESEARCH ARTICLE

\title{
Transpapillary Injection Technique as a Substitute for Palatal Infiltration: A Split-mouth Randomized Clinical Trial
}

\author{
Maria A Sruthi ${ }^{1}$, Mahesh Ramakrishnan ${ }^{2}$
}

\begin{abstract}
Aim and objective:To assess and compare the degree of pain during local anesthesia administration and to evaluate the efficacy of transpapillary injection as a substitute for palatal injection in simple maxillary tooth extractions.

Materials and methods: A split-mouth study was conducted among children aged 7-11 years who required bilateral extraction of maxillary molars. The individuals were subjected to conventional buccal and palatal infiltration for the first extraction. The transpapillary injection was administered for the subsequent extraction in place of palatal administration. $2 \%$ lignocaine with 1:100,000 adrenaline was used for both techniques. Faces pain score scale and visual analog scale were utilized to record pain during injection administration and during the extraction process. A $p<0.05$ was considered to be statistically significant.

Results: The difference in pain scores was observed during injection administration and was found to be statistically significant $(p<0.05)$ whereas pain scores for extraction were insignificant $(p>0.05)$.

Conclusion: The transpapillary injection technique was efficacious in eliminating the pain of the palatal injection technique for maxillary extractions.

Clinical significance: Owing to the results of this study, the transpapillary injection technique can be used as a distinguished substitute for conventional palatal infiltration, thereby lessening the level of distress experienced by a pediatric patient during local anesthesia administration. Keywords: LA administration, Pain during injection, Palatal infiltration, Randomized clinical trial, Transpapillary injection technique. International Journal of Clinical Pediatric Dentistry (2021): 10.5005/jp-journals-10005-2026
\end{abstract}

\section{INTRODUCTION}

Exodontia is a significant part of dental practice and the most common reason for an invasive procedure done in pediatric dental practice. Although the prevalence of dental caries in young children has decreased impressively in recent years, caries continues to affect many children in the general population, some of which result in resorption of the primary teeth and subsequent extraction. Maxillary extraction usually requires administration of local anesthesia in the buccal vestibule followed by a palatal infiltration. Palatal infiltration of local anesthesia is given to anesthetize free nerve endings of nasopalatine or the greater palatine nerves and is often regarded as a painful and difficult procedure. This is because the anesthetic solution causes the separation of firmly bound mucoperiosteum from the underlying bone of the hard palate. Some degree of pain is also produced as a result of a needle penetrating the mucosa. ${ }^{1-3}$

According to Loeser and Treede (2008), pain is characterized as a distressing sensorial or psychological experience arising from definite or probable tissue damage. Generally, dental procedures are stereotypically expressed as grievous and uncomfortable. Pain control is important and necessary for successful pediatric dental care. Due to constant correspondences, dentists tend to assess painless than that of the patient. They associate the symptoms of pain to that of fear and anxiety, especially in children. ${ }^{4}$

Different innovative techniques to reduce this discomfort have been described in the literature with fluctuating degrees of efficacies. These include vapocoolant spray on the palate before injection, ${ }^{2}$ pressure applications on the mucosa by the mirror handle before injecting, ${ }^{3}$ topical anesthetic applications, ${ }^{5}$ withdrawing the needle a little bit before injecting so that solution does not
1,2Department of Pediatric and Preventive Dentistry, Saveetha Dental College and Hospital, Chennai, Tamil Nadu, India

Corresponding Author: Mahesh Ramakrishnan, Department of Pediatric and Preventive Dentistry, Saveetha Dental College and Hospital, Chennai, Tamil Nadu, India, Phone: +91 9840322728, e-mail: maheshpedo@gmail.com

How to cite this article: Sruthi MA, Ramakrishnan M. Transpapillary Injection Technique as a Substitute for Palatal Infiltration: A Splitmouth Randomized Clinical Trial. Int J Clin Pediatr Dent 2021;14(5): 640-643.

Source of support: Nil

Conflict of interest: None

go subperiosteal, ${ }^{6}$ Wand injection system, ${ }^{7}$ nerve stimulation using TENS, ${ }^{8}$ keeping away from palatal injection by using $4 \%$ Articaine $\mathrm{HCl}$ rather than lignocaine $\mathrm{e}^{2,3}$ and intraosseous injection in children. ${ }^{9,10}$

Transpapillary injection is likewise a method for avoiding palatal injection for maxillary extractions. Here, an injection is given through the interdental papilla with the needle inserted from the buccal side to achieve anesthesia on the palatal aspect. The buccal mucosa, including the site of insertion of needle, i.e., the papilla, has already been anesthetized by buccal infiltration thus eliminating the need for a palatal injection. ${ }^{6}$

Therefore, the rationale for the study was to find a feasible alternative to palatal injection which can make the procedure more comfortable for the patient.

The study aimed to analyze and contrast pain during administration of injections through transpapillary and palatal 
infiltration techniques and evaluate their effectiveness for maxillary primary tooth extractions.

\section{Materials and Methods}

A split-mouth study was carried out among children aged 7-11 years in the outpatient block of the Pediatric Dentistry department in a private institution in the month of December 2019 with a sample size of 25 participants. The Institutional Ethical Review Board issued the ethical clearance for the study. The parents/guardians of the participating children were asked to sign an informed consent before the trial by discussing with them the need for the study.

Healthy children aged 7-11 years who required bilateral extraction of maxillary molars after thorough clinical examination and radiographic assessment were included in the study.

Individuals suffering from comorbid conditions, those who were allergic to local anesthetics, those requiring permanent molar extraction, and those who were unable to understand the VAS and FPS were excluded from the study.

All children were subjected to conventional buccal and palatal infiltration techniques to anesthetize the buccal and palatal mucosa for their first maxillary molar extraction and were taken as the control group. The side/quadrant for the intervention was randomly selected. After 1 week, the children were subjected to buccal infiltration followed by a transpapillary injection technique to anesthetize the palatal mucosa for the extraction on the opposite quadrant.

A standard $2.2 \mathrm{~mL}$ cartridge of $2 \%$ lignocaine hydrochloride with 1:100,000 adrenaline was used for both groups; $1.0 \mathrm{~mL}$ solution was injected in the buccal sulcus in both groups and $0.3-0.4 \mathrm{~mL}$ of anesthetic solution was given for the palatal and the transpapillary injection. A 2-minute time period was allowed to pass before giving the palatal or the transpapillary injection after the administration of buccal infiltration. This was done to anesthetize the buccal papilla before needle insertion for the transpapillary injection making it a painless procedure. 1 The same protocol was followed in the control group to eliminate bias. The extraction was carried out after a 3 minutes delay.

The pain of injection and pain experienced during the extraction for both the groups were recorded using the faces pain scales (FPS) and visual analog scale (VAS). For VAS and Wong Bakers FPS, "0" was considered as no pain and "10" being the maximum imaginable pain. Before administering anesthesia, patients were explained how to use these scales at the end of the procedure.

The results were interpreted using SPSS version 23.0. Descriptive statistics were calculated for age and gender. An independent $t$-test was used to analyze pain between the two groups and $p<0.05$ was considered to be statistically significant.

\section{Results}

The study group comprised 14 boys and 11 girls with an age range of 7-11 years (mean $=8.52 \pm 0.99$ ). Results of FPS and VAS scores during injection and extraction procedures were evaluated using an independent $t$-test.

The mean FPS and VAS score for palatal infiltration was 8.40 and 8.20 and the mean scores for transpapillary technique was 5.16 and 4.92 for FPS and VAS, respectively (Table 1). The difference of scores for pain during injection on FPS and VAS were observed to be statistically significant $(p<0.05)$ while that for the extraction procedure were statistically insignificant $(p>0.05)$.

\section{Discussion}

Due to needle phobia, 5-25\% of patients avoid dental treatment. ${ }^{11,12}$ Among the various dental injections that are given routinely for dental procedures; palatal infiltration is regarded as the most painful owing to the tight binding of mucoperiosteum with the bone. ${ }^{2,3}$ Various scales have been used to describe the pain like VAS, FPS, VRS, Shmidt pain index, but a valid evaluation of pain is always subjective and met with difficulties. Donna Wong and Connie Baker instituted the FPS. The scale represents faces and health care professionals are obligated to describe the pain intensity associated with each face. The child is then encouraged to select the face that best describes his/her pain level. ${ }^{13}$ The attributes of pain are well-acknowledged when children are about 7-8 years of age. Hence, self-reporting pain questionnaires' involving visuals and numbers are successful in this age group. Similar to the FPS, the simplest VAS (VPS) is a straight line, $100 \mathrm{~mm}$ in length, and is defined by descriptions at each end associated with pain. The children are requested to indicate a point on the line that portrays their pain at the moment. ${ }^{14}$

Literature is full of materials and techniques proposed by clinicians to overcome the pain of palatal infiltration but none of these has been without some disadvantage. Application of topical sedative (lignocaine, benzocaine eutectic mixture of local anesthesia) on the palate using cotton bud before injection reduces the pain of needle penetration but fails to fight the pain of periosteal separation. Another issue that can be encountered with topical anesthetic is unpleasant to taste and smell for some patients. ${ }^{5}$

Regarding the use of computer-assisted anesthesia and transcutaneous electronic nerve stimulation (TENS) to reduce the pain of palatal infiltration, complex, bulky, and costly equipment is required which is not readily available in most dental set-ups, especially in underdeveloped countries, and even then the results are accurate. ${ }^{7,8}$

Intra-osseous injections have achieved favorable outcomes in endodontic and restorative practices. When used along with a

Table 1: Comparison of FPS and VAS for the two injection techniques

\begin{tabular}{lllll}
\hline Pain score & Injection technique & Total patients & Mean \pm Std mean error & $p$ value (t-test) \\
\hline FPS for injection & Transpapillary injection & 25 & $5.16 \pm 0.64$ & 0.0001 \\
& Palatal injection & 25 & $8.40 \pm 0.82$ & 0.8453 \\
FPS for extraction & Transpapillary injection & 25 & $6.96 \pm 0.73$ & $7.00 \pm 0.71$ \\
& Palatal injection & 25 & $4.92 \pm 0.69$ & 0.0001 \\
VAS for injection & Transpapillary injection & 25 & $8.20 \pm 0.71$ & 0.8507 \\
& Palatal injection & 25 & $6.92 \pm 0.76$ & $6.96 \pm 0.73$ \\
\hline
\end{tabular}


computerized system, it can be regarded as a superior substitute to the conventional injection techniques. ${ }^{9}$

Some studies in the literature show that palatal injections may not be required at all for extraction in the maxilla. ${ }^{11}$ However, a survey conducted in New Zealand and Australia among clinicians regarding the need for palatal injections or not, showed that $91.6 \%$ of practitioners preferred to give palatal injections for maxillary tooth extractions. ${ }^{15}$

Numerous authors have examined the use of $4 \%$ Articaine $\mathrm{HCl}$, in an attempt to eliminate the need for palatal infiltration and were able to exhibit agreeable results. The usage of $1.7 \mathrm{~mL}$ Articaine/ $\mathrm{HCl}$ for palatal anesthesia affords equivalent effectiveness to the classic route. Most dentists are apprehensive with the uneasiness and distress caused by palatal injections and elude its usage unless indispensable. ${ }^{2,3}$

Various authors have used other techniques like combinations of multiple anesthetic solutions and intraligamentary techniques to achieve maxillary anesthesia for tooth extraction and intraligamentary injections. $^{16,17}$

A simple and effective technique that was used in this study to avoid palatal injection was transpapillary injection in which a needle is inserted from the buccal interdental papilla, above the alveolar bone to achieve anesthesia of the palatal papilla and the gingival collar around the tooth. Janjua et al. ${ }^{1}$ proposed a time period of 2 minutes to pass after giving buccal infiltration to allow the buccal papilla to be anesthetized completely before the transpapillary injection thereby reducing the pain.'

The study showed that there was a statistically significant $(p<$ 0.01 ) difference regarding pain experienced during palatal injection and that experienced during transpapillary injection and at the same time, the pain experienced during the extraction procedure was insignificant ( $p=0.8$ ) between our control and study group demonstrating that transpapillary injection technique is equitably efficacious as the conventional technique for palatal anesthesia for maxillary extractions with a less intensity of pain during the administration of anesthesia.

The advantages of this technique, other than reduced pain, are that it does not require any exceptional equipment and its simplicity makes one proficient in its usage. Deposition of vasoconstrictor containing local anesthesia in the gingival collar around the tooth produced better hemostasis than the ordinary palatal injection. This was an added benefit that was observed in this study and was concurrent with a previous study. ${ }^{1}$

The concerns that can be faced during transpapillary injection are that it is slightly tough to administer in posterior teeth especially in the molars and the needle has to be bent to allow administration. Another aspect that can be problematic during anesthesia administration is that the palatal papilla can also be pierced during needle introduction which makes it difficult to achieve anesthesia as the solution leaks from that puncture and this leakage in the oral cavity convey a bitter taste. ${ }^{1}$

The limitations of the study are its small sample size and the subjective description of pain.

\section{Conclusion}

Transpapillary injection is a resourceful and painless alternative to the conventional painful palatal injection for extractions of maxillary primary teeth and should be given whenever feasible so that dental experience can be made as comfortable as possible for the pediatric patient.

\section{Clinical Significance}

Needle penetration, as well as injection pain associated with palatal infiltration, can be reduced with the transpapillary technique. Hemostasis around the gingival collar is also rapidly achieved. This is of utmost significance in pediatric patients who are apprehensive about LA administration.

\section{ACKnOWLedgments}

This research was approved by the Saveetha Institute of Medical and Technical Sciences Review Board. My profound appreciation to Dr Mahesh Ramakrishnan, Saveetha Dental College and Hospital, for his constant encouragement and guide in this study.

\section{References}

1. Janjua OS, Luqman U, Ibrahim MW, et al. Transpapillary versus palatal injection technique for maxillary tooth extractions. J Coll Physicians Surg Pak 2012;22(3):143-146. DOI: 02.2012/JCPSP.143146.

2. Uckan S, Dayangac E, Araz K. Is permanent maxillary tooth removal without palatal injection possible? Oral Surg Oral Med Oral Pathol Oral Radiol Endod 2006;102(6):733-735. DOI: 10.1016/j.tripleo.2005. 12.005.

3. Fan $\mathrm{S}$, Chen $\mathrm{WL}$, Yang $\mathrm{ZH}$, et al. Comparison of the efficiencies of permanent maxillary tooth removal performed with single buccal infiltration versus routine buccal and palatal injection. Oral Surg Oral Med Oral Pathol Oral Radiol Endod 2009;107(3):359-363. DOI: 10.1016/j.tripleo.2008.08.025.

4. Versloot J, Veerkamp JS, Hoogstraten J. Assessment of pain by the child, dentist, and independent observers. Pediatr Dent 2004;26(5):445-449.

5. Al-Melh MA, Andersson L. Comparison of topical anesthetics (EMLA/Oraqix vs. benzocaine) on pain experienced during palatal needle injection. Oral Surg Oral Med Oral Pathol Oral Radiol Endod 2007;103(5):e16-e20. DOI: 10.1016/j.tripleo.2006.11.033.

6. Malamed SF. Handbook of local anesthesia. 5th ed., St Louis: Mosby; 2004. pp. 189-225.

7. Berlin J, Nusstein J, Reader A, et al. Efficacy of articaine and lidocaine in a primary intraligamentary injection administered with a computercontrolled local anesthetic delivery system. Oral Surg Oral Med Oral Pathol Oral Radiol Endod 2005;99(3):361-366. DOI: 10.1016/j. tripleo.2004.11.009.

8. Meechan JG, Gowans AJ, Welbury RR. The use of patient controlled transcutaneous electronic nerve stimulation (TENS) to decrease the discomfort of regional anaesthesia in dentistry: a randomised controlled clinical trial. J Dent 1998;26(5-6):417-420. DOI: 10.1016/ s0300-5712(97)00062-6.

9. Sixou JL, Barbosa-Rogier ME. Efficacy of intraosseous injections of anesthetic in children and adolescents. Oral Surg Oral Med Oral Pathol Oral Radiol Endod 2008;106(2):173-178. DOI: 10.1016/j. tripleo.2007.12.004.

10. Jensen J, Nusstein J, Drum M, et al. Anesthetic efficacy of a repeated intraosseous injection following a primary intraosseous injection. J Endod 2008;34(2):126-130. DOI: 10.1016/j.joen.2007.11.020.

11. Badcock ME, Gordon I, McCullough MJ. A blinded randomized controlled trial comparing lignocaine and placebo administration to the palate for removal of maxillary third molars. Int J Oral Maxillofac Surg 2007;36(12):1177-1182. DOI: 10.1016/j.ijom.2007.06.001.

12. Milgrom P, Coldwell SE, Getz T, et al. Four dimensions of fear of dental injections. J Am Dent Assoc 1997;128(6):756-766. DOI: 10.14219/jada. archive.1997.0301.

13. Wong DL, Baker CM. Pain in children: comparison of assessment scales. Pediatr Nurs 1988;14(1):9-17.

14. McGrath PA, Gillsepie J. Pain assessment in children and adolescents. in Handbook of pain assessment Turk DC, Melzack R, ed. 2nd ed., New York, NY, USA: Gilford Press; 2001. pp. 97-118. 
15. Badcock ME, McCullough MJ. Palatal anaesthesia for the removal of maxillary third molars as practised by oral and maxillofacial surgeons in Australia and New Zealand. Aust Dent J 2007;52(4):329-332. DOI: 10.1111/j.1834-7819.2007.tb00510.x.

16. White JJ, Reader A, Beck M, et al. The periodontal ligament injection: a comparison of the efficacy in human maxillary and mandibular teeth. J Endod 1988;14(10):508-514. DOI: 10.1016/S0099-2399(88) 80109-2.

17. Meechan JG. A comparison of ropivacaine and lidocaine with epinephrine for intraligamentary anesthesia. Oral Surg Oral Med Oral Pathol Oral Radiol Endod 2002;93(4):469-473. DOI: 10.1067/ moe.2002.121390. 